\section{Adiabatic Theorem for Discrete Time Evolution}

\author{
Atushi TANAKA*
}

\section{Department of Physics, Tokyo Metropolitan University, Hachioji, Tokyo 192-0397}

\section{KEYWORDS: adiabatic theorem, quantum map, quantum cir- cuit}

Quantum maps, where the time evolution proceeds along discrete time, describe various important models in studies of quantum chaos. ${ }^{1)}$ Also, quantum maps describe the time evolution of quantum circuits, which play the key role in studies of quantum computation. ${ }^{2)}$ Many studies that concern quantum maps with slow parameters assume that the adiabatic theorem is applicable. ${ }^{3)}$ Although the adiabatic time evolution is the most elementary among studies of quantum dynamics, ${ }^{4}$ ) there have been only a few works on the adiabatic theorem for quantum maps. ${ }^{1)}$ One is a numerical verification by Takami ${ }^{5}$ ) and the other is a heuristic argument by Hogg. ${ }^{6)}$ We note that the proof for slowly modulated Hamiltonians, ${ }^{7)}$ the stationary state of which is described by an eigenvector of a Floquet Hamiltonian, ${ }^{8)}$ is not applicable to quantum maps in general, since the adiabatic parameter can be discontinuous in time for quantum maps.

The aim of this note is to provide a proof of the adiabatic theorem for quantum maps ("discrete adiabatic theorem"), where we employ a discrete time analog of Kato's proof. ${ }^{9-11)}$ Although we will focus on the case where the spectrum of the quantum map is purely discrete and the spectral crossing is absent, various extensions should be straightforward.

We explain the adiabatic limit for quantum maps $\hat{U}(s)$ with a slow parameter $s$. The adiabatic parameter evolves from $s^{\prime}$ to $s^{\prime \prime}$ for $N(>0)$ steps. Let $s_{n}$ be the value of $s$ at the $n$-th step $(0 \leq n \leq N)$, where $s_{0}=s^{\prime}$ and $s_{N}=s^{\prime \prime}$. We assume that the interval $\delta s_{n} \equiv s_{n}-s_{n-1}$ is $O\left(N^{-1}\right)$ as $N \rightarrow \infty$. Also, we assume that $s_{n}$ belongs to a smooth path $C$. We will examine the time evolution induced by $\hat{U}(s)$ with $\left\{s_{n}\right\}_{n}$. The exact time evolution operator $\hat{U}_{n}$ satisfies the recursion relation

$$
\hat{U}_{n}=\hat{U}\left(s_{n}\right) \hat{U}_{n-1}
$$

for $n>0$ and $\hat{U}_{0}=1$.

We introduce assumptions for $\hat{U}(s)$ for our proof. First, $\hat{U}(s)$ is assumed to be unitary, and we assume that its spectrum consists of purely discrete eigenvalues $\left\{e^{i \theta_{j}(s)}\right\}$, where an eigenangle $\theta_{j}(s)$ takes a real value for $s \in C$. The corresponding spectral projections $\left\{\hat{P}_{j}(s)\right\}_{j}$ satisfy $\hat{U}(s) \hat{P}_{j}(s)=$ $e^{i \theta_{j}(s)} \hat{P}_{j}(s)$ and $\hat{P}_{j}(s) \hat{P}_{k}(s)=\delta_{j k} \hat{P}_{j}(s)$. Also, we have the resolution of unity $\sum_{j} \hat{P}_{j}(s)=1$. The second assumption is

$$
z_{j k}(s) \neq 1, \quad \text { where } \quad z_{j k}(s) \equiv \exp \left(-i\left\{\theta_{j}(s)-\theta_{k}(s)\right\}\right),
$$

which corresponds to the nonzero gap condition for eigenenergies. This also implies the absence of a crossing of eigenangles. Hence the dimension of the $j$-th eigenspace is independent of $s$. Finally, we assume that $\hat{P}_{j}(s)$ and $\theta_{j}(s)$ are smooth functions of $s$.

The discrete adiabatic theorem for quantum map $\hat{U}(s)$ is that

$$
\hat{P}_{j}\left(s^{\prime \prime}\right) \hat{U}_{N} \hat{P}_{k}\left(s^{\prime}\right)=\delta_{j k}+O\left(N^{-1}\right)
$$

for $N \rightarrow \infty$. In the following, we will follow a "discrete analog" of the conventional proof of the adiabatic theorem. ${ }^{9,10}$ ) Our proof consists of two parts. One is to introduce the time evolution operator $\hat{W}_{n}$ [eq. (10)] for an interaction picture whose free evolution is the adiabatic time evolution. This is rather straightforward. The other is to examine $\hat{W}_{n}$ to estimate the deviation from the adiabatic time evolution. In the latter part, we will frequently utilize a discrete analog of integration by parts:

$$
\sum_{n^{\prime}=1}^{n}\left(f_{n^{\prime}}-f_{n^{\prime}-1}\right) g_{n^{\prime}}=f_{n} g_{n}-f_{0} g_{1}-\sum_{n^{\prime}=1}^{n-1} f_{n^{\prime}}\left(g_{n^{\prime}+1}-g_{n^{\prime}}\right) .
$$

We introduce two time evolution operators that comprise the adiabatic time evolution. The first part is Kato's geometric evolution operator $\hat{U}_{\mathrm{K}}\left(s, s^{\prime}\right)$, which is assumed to satisfy the intertwining property,

$$
\hat{P}_{j}(s) \hat{U}_{\mathrm{K}}\left(s, s^{\prime}\right)=\hat{U}_{\mathrm{K}}\left(s, s^{\prime}\right) \hat{P}_{j}\left(s^{\prime}\right) .
$$

$\hat{U}_{\mathrm{K}}\left(s, s^{\prime}\right)$ can be expressed by a path ordered integral of an adiabatic "Hamiltonian" $\hat{H}_{\mathrm{K}}(s)$ along a segment of $C$ :

$$
\hat{U}_{\mathrm{K}}\left(s, s^{\prime}\right) \equiv \exp _{\leftarrow}\left\{-i \int_{s^{\prime}}^{s} \hat{H}_{\mathrm{K}}(r) d r\right\},
$$

where $\exp _{\leftarrow}$ represents the path ordered exponential. Here, we employ 9 )

$$
\hat{H}_{\mathrm{K}}(s) \equiv \frac{i}{2} \sum_{j}\left[\frac{\partial \hat{P}_{j}(s)}{\partial s}, \hat{P}_{j}(s)\right],
$$

which satisfies eq. (5) as well as

$$
\hat{P}_{j}(s) \hat{H}_{\mathrm{K}}(s) \hat{P}_{j}(s)=0 \text {. }
$$

The latter equation is convenient to prove the main theorem. The second part of the free evolution contains only the dynamical phase

$$
\hat{U}_{\mathrm{D}, n} \equiv \sum_{j} \hat{P}_{j}\left(s^{\prime}\right) \exp \left\{i \sum_{n^{\prime}=1}^{n} \theta_{j}\left(s_{n^{\prime}}\right)\right\}
$$

for $n>0$ and $\hat{U}_{\mathrm{D}, 0}=1$.

Using the adiabatic time evolution $\hat{U}_{\mathrm{K}}\left(s_{n}, s^{\prime}\right) \hat{U}_{\mathrm{D}, n}$ as a free evolution, we introduce the time evolution operator in the interaction picture:

$$
\hat{W}_{n} \equiv\left\{\hat{U}_{\mathrm{K}}\left(s_{n}, s^{\prime}\right) \hat{U}_{\mathrm{D}, n}\right\}^{\dagger} \hat{U}_{n} .
$$

From the definition of $\hat{U}_{\mathrm{D}, n}$, we have $\hat{W}_{0}=1$. The recursion relation for $\hat{W}_{n}$ is

$$
\hat{W}_{n}=\hat{U}_{W, n} \hat{W}_{n-1}
$$

where

$$
\hat{U}_{W, n} \equiv\left\{\hat{U}_{\mathrm{K}}\left(s_{n}, s^{\prime}\right) \hat{U}_{\mathrm{D}, n}\right\}^{\dagger} \hat{U}\left(s_{n}\right) \hat{U}_{\mathrm{K}}\left(s_{n-1}, s^{\prime}\right) \hat{U}_{\mathrm{D}, n-1} .
$$

To prove the discrete adiabatic theorem [eq. (3)], it suffices to show

$$
\hat{P}_{j}\left(s^{\prime}\right) \hat{W}_{N} \hat{P}_{k}\left(s^{\prime}\right)=\delta_{j k}+\mathcal{O}\left(N^{-1}\right),
$$

which will be shown in the following. We start from a differ- 
ence equation of $\hat{W}_{n}$,

$$
\hat{W}_{n}-\hat{W}_{n-1}=\left(\hat{U}_{W, n}-1\right) \hat{W}_{n-1},
$$

which implies

$$
\hat{W}_{n}=1+\sum_{n^{\prime}=1}^{n}\left(\hat{U}_{W, n^{\prime}}-1\right) \hat{W}_{n^{\prime}-1} .
$$

To apply the discrete analog of integration by parts [eq. (4)], we introduce

$$
\hat{V}_{n} \equiv \sum_{n^{\prime}=1}^{n}\left(\hat{U}_{W, n^{\prime}}-1\right)
$$

for $n>0$ and $\hat{V}_{0}=0$. From eqs. (15) and (4), we obtain

$$
\hat{W}_{n}=1+\hat{V}_{n} \hat{W}_{n-1}-\sum_{n^{\prime}=1}^{n-1} \hat{V}_{n^{\prime}}\left(\hat{U}_{W, n^{\prime}}-1\right) \hat{W}_{n^{\prime}-1} .
$$

In the following, we will show $\hat{U}_{W, n}-1=O\left(N^{-1}\right)$ and $\hat{V}_{n}=$ $O\left(N^{-1}\right)$, which imply eq. (13).

We examine $\hat{P}_{j}\left(s^{\prime}\right)\left(\hat{U}_{W, n}-1\right) \hat{P}_{k}\left(s^{\prime}\right)$ for $j \neq k$ :

$$
\hat{P}_{j}\left(s^{\prime}\right)\left(\hat{U}_{W, n}-1\right) \hat{P}_{k}\left(s^{\prime}\right)=Z_{n-1, j k} \hat{R}_{n, j k},
$$

where

$$
Z_{n, j k} \equiv \exp \left\{-i \sum_{n^{\prime}=1}^{n}\left[\theta_{j}\left(s_{n^{\prime}}\right)-\theta_{k}\left(s_{n^{\prime}}\right)\right]\right\}
$$

and

$$
\hat{R}_{n, j k} \equiv\left\{\hat{U}_{\mathrm{K}}\left(s_{n}, s^{\prime}\right)\right\}^{\dagger} \hat{P}_{j}\left(s_{n}\right) \hat{P}_{k}\left(s_{n-1}\right) \hat{U}_{\mathrm{K}}\left(s_{n-1}, s^{\prime}\right) .
$$

From the smoothness of $\hat{P}_{j}(s)$, we have

$$
\hat{P}_{j}\left(s_{n}\right)=\hat{P}_{j}\left(s_{n-1}\right)+\hat{P}_{j}^{\prime}\left(s_{n-1}\right) \delta s_{n}+O\left(\left(\delta s_{n}\right)^{2}\right),
$$

which implies

$$
\hat{R}_{n, j k}=O\left(N^{-1}\right) \text { for } j \neq k .
$$

Hence, we obtain $\hat{P}_{j}\left(s^{\prime}\right)\left(\hat{U}_{W, n}-1\right) \hat{P}_{k}\left(s^{\prime}\right)=O\left(N^{-1}\right)$. On the other hand, the "diagonal" part of $\hat{U}_{W, n}-1$ is

$$
\begin{aligned}
& \hat{P}_{j}\left(s^{\prime}\right)\left(\hat{U}_{W, n}-1\right) \hat{P}_{j}\left(s^{\prime}\right) \\
& =\left\{\hat{U}_{\mathrm{K}}\left(s_{n}, s^{\prime}\right)\right\}^{\dagger} \hat{P}_{j}\left(s_{n}\right)\left\{1-\hat{U}_{\mathrm{K}}\left(s_{n}, s_{n-1}\right)\right\} \hat{P}_{j}\left(s_{n-1}\right) \hat{U}_{\mathrm{K}}\left(s_{n-1}, s^{\prime}\right) .
\end{aligned}
$$

From eqs. (6) and (8), we have $1-\hat{U}_{\mathrm{K}}\left(s_{n}, s_{n-1}\right)=O\left(\left(\delta s_{n}\right)^{2}\right)$. Hence, we conclude $\hat{P}_{j}\left(s^{\prime}\right)\left(\hat{U}_{W, n}-1\right) \hat{P}_{j}\left(s^{\prime}\right)=O\left(N^{-2}\right)$, which is much smaller than the off-diagonal components of $\left(\hat{U}_{W, n}-1\right)$.

Next, we examine $\hat{V}_{n}$. The diagonal part is $\hat{P}_{j}\left(s^{\prime}\right) \hat{V}_{n} \hat{P}_{j}\left(s^{\prime}\right)=$ $\sum_{n^{\prime}=1}^{n} \hat{P}_{j}\left(s^{\prime}\right)\left(\hat{U}_{W, n^{\prime}}-1\right) \hat{P}_{j}\left(s^{\prime}\right)=O\left(n / N^{2}\right)$. Hence, we have $\hat{P}_{j}\left(s^{\prime}\right) \hat{V}_{n} \hat{P}_{j}\left(s^{\prime}\right)=O\left(N^{-1}\right)$ for $0<n \leq N$. On the other hand, our estimation of the off-diagonal part requires the destructive quantum interference effect induced by the dynamical phase factors to be taken into account. Namely, our task is to examine the following oscillatory summation,

$$
\hat{P}_{j}\left(s^{\prime}\right) \hat{V}_{n} \hat{P}_{k}\left(s^{\prime}\right)=\sum_{n^{\prime}=1}^{n} Z_{n^{\prime}-1, j k} \hat{R}_{n^{\prime}, j k},
$$

for $j \neq k$. To apply eq. (4) to eq. (24), we examine the difference of $Z_{n, j k}$ :

$$
Z_{n, j k}-Z_{n-1, j k}=Z_{n-1, j k}\left\{z_{j k}\left(s_{n}\right)-1\right\}
$$

[see eqs. (2) and (19)]. From the noncrossing condition for the eigenangle [eq. (2)], we have

$$
Z_{n-1, j k}=\frac{Z_{n, j k}-Z_{n-1, j k}}{z_{j k}\left(s_{n}\right)-1} .
$$

From eq. (24), we have

$$
\begin{aligned}
& \hat{P}_{j}\left(s^{\prime}\right) \hat{V}_{n} \hat{P}_{k}\left(s^{\prime}\right) \\
& =\frac{Z_{n, j k} \hat{R}_{n, j k}}{z_{j k}\left(s_{n}\right)-1}-\frac{\hat{R}_{1, j k}}{z_{j k}\left(s_{1}\right)-1}+\sum_{n^{\prime}=1}^{n-1} Z_{n^{\prime}, j k} \hat{R}_{n^{\prime}, j k}^{(2)},
\end{aligned}
$$

where

$$
\hat{R}_{n, j k}^{(2)} \equiv \frac{\hat{R}_{n+1, j k}}{z_{j k}\left(s_{n+1}\right)-1}-\frac{\hat{R}_{n, j k}}{z_{j k}\left(s_{n}\right)-1} .
$$

The first two terms on the right-hand side of eq. (27) are $O\left(N^{-1}\right)$ from eqs. (22) and (2). It is straightforward to see that $R_{n}^{(2)}=O\left(N^{-2}\right)$ from eq. (2) and the smoothness of $\hat{P}_{j}(s)$ and $\theta_{j}(s)$. These estimations imply $\hat{P}_{j}\left(s^{\prime}\right) \hat{V}_{n} \hat{P}_{k}\left(s^{\prime}\right)=O\left(N^{-1}\right)$ for $j \neq k$. Thus, we have confirmed eq. (13). This completes the proof of the discrete adiabatic theorem.

NOTE ADDED: After the completion of this work, Professor Alain Joye kindly informed me of an earlier work by A. Dranov, J. Kellendonk and R. Seiler [J. Math. Phys. 39 (1998) 1340]. Their work is an adaptation of ref. 11, which is a thorough extension of Kato's proof in ref. 9, to discrete time evolution. In contrast to this, the proof of this Short Note is a descendent of ref. 10, which is a simplification of ref. 9 for systems whose spectrum is purely discrete. It seems that a simpler argument may yet be of some value to compare the discrete and continuous time settings.

Acknowledgments I wish to thank Toshiya Takami for discussion. This research was supported by the Japan Ministry of Education, Culture, Sports, Science and Technology under Grant number 22540396.

1) M. Berry, N. Balazs, M. Tabor, and A. Voros: Ann. Phys. (N.Y.) 122 (1979) 26.

2) M. A. Nielsen and I. L. Chuang: Quantum Computation and Quantum Information (Cambridge University Press, Cambridge, 2000)

3) For example, P. Cabauy and P. Benioff: Phys. Rev. A 68 (2003) 032315; A. Tanaka and M. Miyamoto: Phys. Rev. Lett. 98 (2007) 160407.

4) M. Born and V. Fock: Z. Phys. A 51 (1928) 165.

5) T. Takami: private communication (1995).

6) T. Hogg: Phys. Rev. A 67 (2003) 022314

7) R. H. Young and W. J. Deal Jr.: J. Math. Phys. 11 (1970) 3298.

8) J. H. Shirley: Phys. Rev. 138 (1965) B979.

9) T. Kato: J. Phys. Soc. Jpn. 5 (1950) 435.

10) A. Messiah: Méchanique Quantique (Dunod, Paris, 1959) Chap. 17.

11) J. E. Avron, R. Seiler, and L. Yaffe: Comm. Math. Phys. 110 (1987) 33. 